\title{
Normal-superfluid interface for polarized fermion gases
}

\author{
Bert Van Schaeybroeck and Achilleas Lazarides \\ Instituut voor Theoretische Fysica, Katholieke Universiteit Leuven, Celestijnenlaan 200 D, B-3001 Leuven, Belgium
}

(Received 23 July 2008; published 8 May 2009)

\begin{abstract}
Recent experiments on imbalanced fermion gases have proved the existence of a sharp interface between a superfluid and a normal phase. We show that, at the lowest experimental temperatures, a temperature difference between normal (N) and superfluid (SF) phases can appear as a consequence of the blocking of energy transfer across the interface. Such blocking is a consequence of the existence of a SF gap, which causes low-energy normal particles to be reflected from the N-SF interface. Our quantitative analysis is based on the HartreeFock-Bogoliubov-de Gennes formalism, which allows us to give analytical expressions for the thermodynamic properties and characterize the possible interface scattering regimes, including the case of unequal masses. Our central result is that the thermal conductivity is exponentially small at the lowest experimental temperatures.
\end{abstract}

DOI: 10.1103/PhysRevA.79.053612

PACS number(s): 03.75.Ss, 03.75.Hh, 68.03.Cd, 68.08.Bc

\section{INTRODUCTION}

By mixing fermion species of unequal particle number, recent experiments have revealed many aspects of the exciting physics of imbalanced fermion gases [1-7]. In these experiments, a trap is loaded with an ultracold spin mixture consisting of two lithium hyperfine states. The existence of an easily accessible Feshbach resonance allows the tuning of the interspecies interactions with great accuracy so that the entire BEC-BCS crossover with number imbalance may be explored [8]. The BCS regime involves a small and negative $s$-wave scattering length, with the fermions forming weakly bound Cooper pairs. On the other hand, in the BEC regime, where the scattering length is positive, the particles form tightly bound molecular pairs forming a condensate [8-11]. In between lies the unitary regime, characterized by a diverging scattering length (signifying the appearance of a bound state), where particles are strongly interacting. The divergence of the scattering length implies that the system is universal, in the sense that only a single length scale characterizes it.

Much work has been done in an attempt to understand the ground-state properties of the imbalanced clouds; however, despite theoretical advances [12-23], some problems remain unresolved. The MIT data [3-7] are in good agreement with Monte Carlo (MC) simulations [22] and renormalizationgroup (RG) predictions [15], which point to the existence of a three-shell structure consisting of a superfluid (SF) core phase, a partially polarized and a fully polarized normal $(\mathrm{N})$ phase. From these experiments, the homogeneous phase diagram has been determined and is in agreement with both the MC and RG results. On the other hand, the results of the Rice group's experiments [1,2] are less well understood. For example, they find that superfluidity does not break down at high polarizations, which is incompatible with the results of the MIT experiments and thus also in contradiction to theoretical predictions of a critical polarization, above which superfluidity breaks down. Since both the MIT phase diagram and the MC and RG predictions were obtained under the assumption that the local-density approximation (LDA) holds, and since the Rice experiments involve smaller traps, it is likely that the answer to the riddle lies in the breakdown of LDA (in other words, finite-size effects); indeed, taking as an ansatz a two-shell structure, the Rice data can be recovered by using a N-SF interface tension as a fitting parameter $[19,20]$.

For our purposes, it is sufficient to say that the MIT experiments are well-described by theory, and furnish direct evidence that a N-SF interface exists, separating an unpolarized SF from a partially polarized $\mathrm{N}$.

In this paper we argue that the N-SF interface may block the thermal equilibration process between the $\mathrm{N}$ and $\mathrm{SF}$ phases; one consequence of this will be to cause a temperature imbalance to appear across the interface. Incorporating such temperature difference in the existing models may provide the key to a better understanding of the experiments. We present a detailed study of the scattering off the N-SF interface within the Hartree-Fock-Bogoliubov-de Gennes (HF$\mathrm{BdG}$ ) formalism, followed by a calculation of the heat conductivity across the interface. We find that this conductivity drops exponentially fast with increasing $\Delta / k_{B} T$ with $\Delta$ the SF gap and $T$ the temperature. Our model predicts the conductivity to be vanishingly small for the experiments at unitarity. A summary of our results was published earlier in Ref. [24].

The paper is structured as follows. We start off in Sec. II by discussing the bulk physics of imbalanced fermion gases. This is done using a mean-field Hamiltonian which incorporates both the gap formation and the Hartree-Fock terms. We present analytical results for the equations of state which enable us to write down the condition for coexistence between the N and the SF phases. Then, in Sec. III, we discuss in detail how particles scatter on a N-SF interface according to the Hartree-Fock-Bogoliubov-de Gennes formalism. We find a rich variety of interfacial scattering processes, depending on the energy and momentum of the incident particle. The implications of this scattering are outlined in Sec. IV, where we obtain the thermal conductivity exactly and with a good analytical approximation. Finally in Sec. V we calculate that for the experiments at lowest temperatures, the typical timescale associated with the equilibration of a temperature difference across the interface, is of order seconds and therefore comparable with the trap lifetime. 


\section{BULK PHYSICS AND COEXISTENCE}

\section{A. Bulk phases}

Fermion mixtures in the BEC-BCS crossover are the subject of a very active research field. Although some theoretical models give accurate results for the BEC and/or BCS regimes, no model yet allows an analytical, quantitative description across the entire crossover. In the following, we use the so-called one-channel model at zero temperature; this is a straightforward generalization of the BCS model to arbitrarily strong coupling and is widely used in the literature, as it is known to embody most qualitative features of the BEC$\mathrm{BCS}$ crossover for the currently experimentally relevant Feshbach resonances. Interactions between fermions and the bosonic bound states may be explicitly incorporated within a two-channel model [9].

We start off by deriving the Hartree-Fock-Bogoliubov-de Gennes equations. We then focus on the physics of an interface separating two semi-infinite phases: a SF and a polarized N. We write down the equations of state in these phases, and use them to find the condition for two-phase coexistence. In this section, we extend the work reported in Ref. [24] by including the $\mathrm{HF}$ term into the $\mathrm{BdG}$ equations and treating the case of unequal species masses.

The system under consideration consists of two fermionic species $\uparrow$ and $\downarrow$ with masses $m_{i}$ and chemical potentials $\mu_{i}$ for $i=\uparrow, \downarrow$ and which we assume to be trapped by the external potential $V_{i}(\mathbf{r})$. The intraspecies interactions are via $p$-wave scattering which can be safely ignored at low temperatures. On the other hand, the interactions between the two spin species are taken to be contact interactions characterized by the coupling constant $G=4 \pi \hbar^{2} a / m_{+}<0$ with $m_{+}$ $\equiv 2\left(m_{\uparrow}^{-1}+m^{-1}\right)^{-1}$ and $a$ the $s$-wave scattering length. Within a generalized HF approximation, one may work with the following Hamiltonian [25,26]:

$$
\begin{aligned}
\hat{H}= & \int_{V} d \mathbf{r}\left[\hat{\Psi}_{\uparrow}^{\dagger}(\mathbf{r}) \mathcal{H}_{\uparrow}(\mathbf{r}) \hat{\Psi}_{\uparrow}(\mathbf{r})+\hat{\Psi}_{\downarrow}^{\dagger}(\mathbf{r}) \mathcal{H}_{\downarrow}(\mathbf{r}) \hat{\Psi}_{\downarrow}(\mathbf{r})\right. \\
& \left.+\Delta(\mathbf{r}) \hat{\Psi}_{\uparrow}^{\dagger}(\mathbf{r}) \hat{\Psi}_{\downarrow}^{\dagger}(\mathbf{r})+\Delta^{*}(\mathbf{r}) \hat{\Psi}_{\downarrow}(\mathbf{r}) \hat{\Psi}_{\uparrow}(\mathbf{r})\right],
\end{aligned}
$$

where $\mathcal{H}_{i} \equiv-\hbar^{2} \nabla^{2} /\left(2 m_{i}\right)-\mu_{i}+V_{i}(\mathbf{r})+U_{i}(\mathbf{r})$, the gap $\Delta=$ $-G\left\langle\hat{\Psi}_{\uparrow}(\mathbf{r}) \hat{\Psi}_{\downarrow}(\mathbf{r})\right\rangle$, and the HF terms $U_{\uparrow}(\mathbf{r})=G\left\langle\hat{\Psi}_{\downarrow}^{\dagger}(\mathbf{r}) \hat{\Psi}_{\downarrow}(\mathbf{r})\right\rangle$ and $U_{\downarrow}(\mathbf{r})=G\left\langle\hat{\Psi}_{\uparrow}^{\dagger}(\mathbf{r}) \hat{\Psi}_{\uparrow}(\mathbf{r})\right\rangle$. As usual, one diagonalizes Hamiltonian (1) by a Bogoliubov transformation, i.e., by writing the operators $\Psi_{i}$ in terms of fermionic quasiparticle operators $\hat{c}$,

$$
\begin{aligned}
& \hat{\Psi}_{\uparrow}(\mathbf{r}, t)=\sum_{\ell} e^{-i E_{\ell} t / \hbar}\left[u_{\ell, \uparrow}(\mathbf{r}) \hat{c}_{\ell, \uparrow}-v_{\ell, \uparrow}(\mathbf{r}) \hat{c}_{\ell, \downarrow}^{\dagger}\right], \\
& \hat{\Psi}_{\downarrow}(\mathbf{r}, t)=\sum_{\ell} e^{-i E_{\ell} t / \hbar}\left[u_{\ell, \downarrow}(\mathbf{r}) \hat{c}_{\ell, \downarrow}-v_{\ell, \downarrow}(\mathbf{r}) \hat{c}_{\ell, \uparrow}^{\dagger}\right],
\end{aligned}
$$

where $u_{\ell, i}$ and $v_{\ell, i}$ are the quasiparticle wave functions, $\left\{\hat{c}_{\ell, i}, \hat{c}_{\ell^{\prime}, j}\right\}=0$ and $\left\{\hat{c}_{\ell, i}^{\dagger}, \hat{c}_{\ell^{\prime}, j}\right\}=\delta_{\ell, \ell^{\prime}} \delta_{i, j}$. Then, if under this transformation Hamiltonian (1) is diagonalized, the relations $\left[\hat{H}, \hat{c}_{\ell, i}\right]=-E_{\ell} \hat{c}_{\ell, i}$ and $\left[\hat{H}, \hat{c}_{\ell, i}^{\dagger}\right]=E_{n} \hat{c}_{\ell, i}^{\dagger}$ must hold such that one straightforwardly finds the four Hartree-Fock-
Bogoliubov-de Gennes, or Blonder-Tinkham-Klapwijk equations [25,27],

$$
\begin{gathered}
\mathcal{H}_{\uparrow} u_{\ell, \uparrow}+\Delta v_{\ell, \downarrow}=E_{\ell} u_{\ell, \uparrow}, \\
\Delta^{*} u_{\ell, \uparrow}-\mathcal{H}_{\downarrow} v_{\ell, \downarrow}=E_{\ell} v_{\ell, \downarrow}, \\
\mathcal{H}_{\downarrow} u_{\ell, \downarrow}+\Delta v_{\ell, \uparrow}=E_{\ell} u_{\ell, \downarrow}, \\
\Delta^{*} u_{\ell, \downarrow}-\mathcal{H}_{\uparrow} v_{\ell, \uparrow}=E_{\ell} v_{\ell, \uparrow} .
\end{gathered}
$$

The wave functions $u_{\ell, \uparrow}$ and $v_{\ell, \downarrow}$ which appear in the first two equations are not coupled to the wave functions $u_{\ell, \downarrow}$ and $v_{\ell, \uparrow}$ of the last two equations so that, apart from normalization, they can be treated separately.

In the rest of this section, we will be concerned with the study of a homogeneous system of two fermion species at fixed chemical potentials $\mu_{\uparrow}$ and $\mu_{\downarrow}$ and with no external potential $V_{\uparrow}=V_{\downarrow}=0$. Furthermore we treat both the case of a $\mathrm{N}$ and a SF phase.

At zero temperature and for negative scattering length, there always exists a (meta-)stable state with $\Delta=\Delta^{*} \neq 0$ and for which both species densities are equal; we call this state the BCS state or SF phase. For this state, we define now the effective chemical-potential imbalance $h_{S}$ and the average effective chemical potential $\mu_{S}$ as follows:

$$
\begin{gathered}
\mu_{S} \equiv \frac{\mu_{\uparrow}+\mu_{\downarrow}}{2}-U_{S}, \\
h_{S} \equiv \frac{\mu_{\uparrow}-\mu_{\downarrow}}{2} .
\end{gathered}
$$

Without loss of generality, we further assume that $h_{S}>0$. Note that in the SF phase, both spin species have equal densities which implies equal HF potentials $U_{S}=U_{\uparrow}=U_{\downarrow}$ for the spin species. One can then find the ground-state energy of the homogeneous SF system as a function of $\mu_{\uparrow}, \mu_{\downarrow}$, and $a$. Solving the HFBdG Eq. (2), and minimizing the ground-state energy with respect to the variables $\Delta$ and $U_{S}$, the gap and number equations at zero temperature are [28]:

$$
\begin{aligned}
& 1=-\frac{G}{2} \int \frac{d^{3} \mathbf{k}}{(2 \pi)^{3}}\left[\frac{1}{\sqrt{\left(\varepsilon_{\mathbf{k}}-\mu_{S}\right)^{2}+\Delta^{2}}}-\frac{1}{\varepsilon_{\mathbf{k}}}\right], \\
& U_{S}=-\frac{G}{2} \int \frac{d^{3} \mathbf{k}}{(2 \pi)^{3}}\left[1-\frac{\varepsilon_{\mathbf{k}}-\mu_{S}}{\sqrt{\left(\varepsilon_{\mathbf{k}}-\mu_{S}\right)^{2}+\Delta^{2}}}\right],
\end{aligned}
$$

where $\varepsilon_{\mathbf{k}} \equiv \hbar^{2} \mathbf{k}^{2} /\left(2 m_{+}\right)$. Note that the above equations are coupled by the term $U_{S}$ which appears in the definition of $\mu_{S}$. The ground-state grand potential per unit volume in terms of $\Delta$ and $\mu_{S}$ is

$$
\Omega_{S}=\int \frac{d^{3} \mathbf{k}}{(2 \pi)^{3}}\left[\varepsilon_{\mathbf{k}}-\mu_{S}-\frac{\Delta^{2} / 2+\left(\varepsilon_{\mathbf{k}}-\mu_{S}\right)^{2}}{\sqrt{\left(\varepsilon_{\mathbf{k}}-\mu_{S}\right)^{2}+\Delta^{2}}}\right] .
$$

We are mostly interested in the BCS side of the BEC-BCS crossover where the effective chemical potentials $\mu_{i}-U_{i}$ and $\mu_{S}$ can be taken as positive. Performing the integrals over the three-dimensional wave vectors leads to the analytical ex- 
pressions for the gap, the HF potential and the grand potential per unit volume [29-32],

$$
\begin{gathered}
\epsilon_{a}=-2 \mu_{S}\left[P_{1 / 2}(\eta)\right]^{2} / \eta \\
U_{S}=\mu_{S}\left[1-\eta^{-1} P_{3 / 2}(\eta) / P_{1 / 2}(\eta)\right], \\
\Omega_{S}=-\frac{\left(m_{+}\right)^{3 / 2} \epsilon_{a}^{5 / 2} \eta^{2}}{80 \pi \hbar^{3}\left|P_{1 / 2}(\eta)\right|^{5}}\left[\left(\eta^{-2}-5\right) P_{1 / 2}(\eta)+4 \eta^{-1} P_{3 / 2}(\eta)\right] .
\end{gathered}
$$

with $P$ the Legendre function, $\eta \equiv-1 / \sqrt{1+\left(\Delta / \mu_{S}\right)^{2}}$ and we introduced the energy scale which is set by the scattering length $\epsilon_{a} \equiv \hbar^{2} /\left(m_{+} a^{2}\right)$. Expression (6c) allows to write the grand potential in terms of $\Delta / \epsilon_{a}$; indeed, for each value thereof, one can find $\eta$ by solving the relation,

$$
1=\eta^{2}+4\left(\Delta / \epsilon_{a}\right)^{2}\left[P_{1 / 2}(\eta)\right]^{4},
$$

such that the grand potential (6c) is fully determined by $\Delta / \epsilon_{a}$ only.

For the normal state, one finds the following expressions for the grand potential per unit volume of the ground state and for the HF potentials:

$$
\begin{gathered}
\Omega_{N}=-\frac{2 \sqrt{2}}{15 \pi^{2} \hbar^{3}}\left[m_{\uparrow}^{3 / 2}\left(\mu_{\uparrow}-U_{\uparrow}\right)^{5 / 2}+m_{\downarrow}^{3 / 2}\left(\mu_{\downarrow}-U_{\downarrow}\right)^{5 / 2}\right], \\
\frac{U_{\uparrow}}{\epsilon_{a}}=-\frac{4 \sqrt{2}}{3 \pi}\left(\frac{m_{\downarrow}}{m_{+}}\right)^{3 / 2}\left(\frac{\mu_{\downarrow}-U_{\downarrow}}{\epsilon_{a}}\right)^{3 / 2}, \\
\frac{U_{\downarrow}}{\epsilon_{a}}=-\frac{4 \sqrt{2}}{3 \pi}\left(\frac{m_{\uparrow}}{m_{+}}\right)^{3 / 2}\left(\frac{\mu_{\uparrow}-U_{\uparrow}}{\epsilon_{a}}\right)^{3 / 2} .
\end{gathered}
$$

At fixed chemical potentials, the attractive interactions induce an increased normal-state density and a decreased ground-state grand potential. Equations $(8 b)$ and $(8 c)$ are coupled in a nontrivial manner and can be solved numerically to find $U_{\uparrow}$ and $U_{\downarrow}$.

\section{B. Bulk two-phase coexistence}

Before writing down the N-SF coexistence condition, we explain how a variation in the chemical-potential imbalance between the spin species may change the ground state. A small imbalance (or polarization) gives rise to a SF state while a large polarization leads to a N state. Intuitively this is expected since it is known from BCS theory that pair formation is energetically favorable for particles with opposite momenta. By shifting the Fermi energies, an energy cost must be paid which is associated with the matching of the Fermi levels so as to enable pair formation; when this energy cost is too high, the $\mathrm{N}$ state prevails.

In a trapped imbalanced fermion system, both the $\mathrm{N}$ and the SF phase may be present, bounded by an interface. Using a sufficiently large number of particles, a local-density approximation is justified there and amounts to using an effective local chemical potential $\mu_{i}(\mathbf{r})=\mu_{i}^{0}-V_{i}(\mathbf{r})$ with $\mu_{i}^{0}$ the chemical potential at the trap center and $i=\uparrow, \downarrow$ [5,19,33-37]. Since in experiments both spin species feel the same trapping potential, the imbalance $\mu_{\uparrow}(\mathbf{r})-\mu_{\downarrow}(\mathbf{r})$ is constant throughout the trap. On the other hand, the average chemical potential $\left[\mu_{\uparrow}(\mathbf{r})+\mu_{\uparrow}(\mathbf{r})\right] / 2$ varies: it is large at the center of the trap and decreases toward the trap boundary. The ground-state energy of the SF increases with increasing value of the gap; as seen from Eq. (6a), the gap value is proportional to the average chemical potential $\mu_{S}$ and independent of $h_{S}$. Therefore, if present, the SF phase will be at the trap center where $\mu_{S}$ is maximal. Moving radially outward, two-phase coexistence of the $\mathrm{N}$ and SF phase occurs at the position where there is a balance between the energy gained by creating, on the one hand, a gap in the SF, and, on the other hand, a density difference in the $\mathrm{N}$ phase.

To establish now the condition for coexistence between the $\mathrm{N}$ and the SF phase, recall that we have a system containing two fermion species $i=\uparrow, \downarrow$ with masses $m_{i}$ and chemical potentials $\mu_{i}$ for which interactions are characterized by a negative scattering length $a$. We aim to find the coexistence condition as a function of the parameters $a, \mu_{\uparrow}$, and $\mu_{\downarrow}$. The SF state is fully described by the parameters $\Delta$ and $U_{S}$, which can be extracted from Eqs. (6a), (6b), and (7). The normal state, on the other hand, is characterized by $U_{\uparrow}$ and $U_{\downarrow}$ which are solved for using Eqs. (8b) and (8c).

The coexistence condition obtained by equating the grand potentials of Eqs. (6c) and (8a) is

$$
\begin{aligned}
& \frac{3 \sqrt{2} \pi \eta^{2}}{64\left|P_{1 / 2}(\eta)\right|^{5}}\left[\left(\eta^{-2}-5\right) P_{1 / 2}(\eta)+4 \eta^{-1} P_{3 / 2}(\eta)\right] \\
& =\left(\frac{m_{\uparrow}}{m_{+}}\right)^{3 / 2}\left(\frac{\mu_{\uparrow}-U_{\uparrow}}{\epsilon_{a}}\right)^{5 / 2}+\left(\frac{m_{\downarrow}}{m_{+}}\right)^{3 / 2}\left(\frac{\mu_{\downarrow}-U_{\downarrow}}{\epsilon_{a}}\right)^{5 / 2} .
\end{aligned}
$$

By condition (9) one can find the surface of coexistence in the space of the parameters $\left(\mu_{\uparrow}, \mu_{\downarrow}, a\right)$.

For one specific case it is easy to relate the gap value to the chemical potentials at coexistence; this occurs in case of equal masses $m_{+}=m_{\uparrow}=m_{\downarrow}$ without incorporation of the HF term. The energy scale $\epsilon_{a}$ can then be eliminated and by a numerical analysis, one finds to a good approximation the Clogston relation, originally derived for superconductors in a magnetic field [38],

$$
\Delta=\sqrt{2} h_{S} .
$$

The Clogston limit is exact in the BCS limit, when $h_{S} \ll \mu_{S}$.

Lastly, it is common to write the scattering length $a$ in units of $1 / k_{F}$, the latter defined by the relations $E_{F}=k_{B} T_{F}$ $=\hbar^{2} k_{F}^{2} /\left(2 m_{+}\right)=\hbar^{2}\left[3 \pi^{2} n_{S}\right]^{2 / 3} /\left(2 m_{+}\right)$; we find that in the SF,

$$
k_{F} a=\left[\frac{3 \pi}{4} \frac{\left(P_{3 / 2}(\eta) / P_{1 / 2}(\eta)-\eta\right)}{P_{1 / 2}^{2}(\eta)}\right]^{1 / 3} .
$$

\section{INTERFACE SCATTERING}

\section{A. Bulk particles}

In this section, we explain in full detail how particles scatter from a N-SF interface for imbalanced fermion gases. 


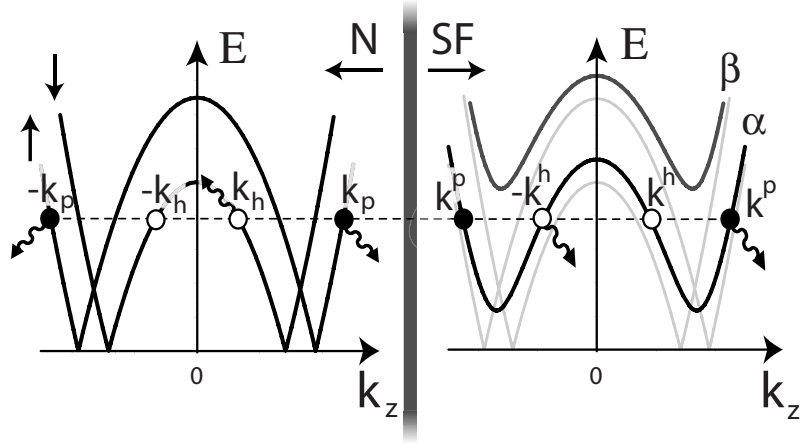

FIG. 1. The N-SF interface (thick vertical line) with the spectra of the species $\uparrow$ and $\downarrow$ on the $\mathrm{N}$ side and the gapped $\alpha, \beta$ spectra in the SF. The long-dashed line cuts the spectra at particlelike (filled dots) and holelike (empty dots) quasiparticle states, all having the same energy. The minimal values of the $\alpha$ and the $\beta$ spectrum are $2\left(\sqrt{\tilde{m}} \Delta-\varepsilon_{\alpha, \beta}^{0}\right) /(\tilde{m}+1)$ where $\varepsilon_{\alpha, \beta}^{0} \equiv\left[ \pm h_{S}(1+\tilde{m})+\mu_{S}(1-\tilde{m})\right] / 2$. An incident particle of species $\uparrow$ (curly arrow) with momentum $k_{p}$ and energy $E$ can have up to four scattering channels: the Andreev reflected $k_{h}$ hole, the specularly reflected $-k_{p}$ particle and the transmitted holelike $-k^{h}$ and particlelike $k^{p}$ states.

By taking a simple model for the interface, we describe the different scattering regimes and we give the transmission coefficients for scattering across the interface; the latter will be used for calculating transport quantities in Sec. IV.

To model the N-SF interface, we start with a geometry wherein the $z=0$ plane separates the $\mathrm{N}$ phase at $z<0$ from the $\mathrm{SF}$ phase at $z>0$ or

$$
\Delta(\mathbf{r})=\Theta(z) \Delta,
$$

with $\Theta$ the Heaviside function. This approximation was also used by Andreev [39] for studying the normalsuperconductor interface.

A particle of energy $E$ incident on the interface may scatter to states which have the same energy and these are deduced from the spectra in the $\mathrm{SF}$ and in the $\mathrm{N}$ phase. In the $\mathrm{N}$ phase, we have for species $i=\uparrow, \downarrow$,

$$
E_{i}=\left|\frac{\hbar^{2} \mathbf{k}^{2}}{2 m_{i}}-\mu_{i}+U_{i}\right| \text {. }
$$

We depict with full lines this spectrum in the left panel of Fig. 1 where one recognizes two approximately linear energy branches $\uparrow$ and $\downarrow$ near the (unequal) Fermi surfaces. In the $\mathrm{SF}$ on the other hand, one finds using the HFBdG equations, an $\alpha$ and a $\beta$ spectrum,

$$
E_{\alpha, \beta}=\mp h_{S} \pm \frac{\hbar^{2} \mathbf{k}^{2}}{2 m_{-}}+\sqrt{\left(\frac{\hbar^{2} \mathbf{k}^{2}}{2 m_{+}}-\mu_{S}\right)^{2}+\Delta^{2}},
$$

where $m_{ \pm}=2\left(m_{\uparrow}^{-1} \pm m^{-1}\right)^{-1}$. These gapped spectra are depicted in the right panel of Fig. 1. The $\alpha$ branch is composed of particles of phase $\uparrow$ and holes of phase $\downarrow$ and vice versa for the $\beta$ spectrum. One can find that the minimal values attained by the $\alpha$ and $\beta$ spectra are $2\left(\sqrt{\widetilde{m}} \Delta-\varepsilon_{\alpha, \beta}^{0}\right) /(\widetilde{m}+1)$ where $\varepsilon_{\alpha, \beta}^{0} \equiv\left[ \pm h_{S}(1+\widetilde{m})+\mu_{S}(1-\widetilde{m})\right] / 2$ with $\quad \widetilde{m} \equiv m_{\downarrow} / m_{\uparrow}$, whence the lowest spectrum touches the $E=0$ axis or becomes ungapped under the condition that $\sqrt{\tilde{m}} \Delta<\varepsilon_{\alpha}^{0}$. We as- sume a gapped spectrum. Since the gap function is constant in both the $\mathrm{N}$ and the SF phase, we can decompose the quasiparticle wave functions $u_{\uparrow}$ and $v_{\downarrow}$, associated with the $\alpha$ branch, into their Fourier components,

$$
\begin{gathered}
\left(\begin{array}{c}
u_{\uparrow}^{n} \\
v_{\downarrow}^{n}
\end{array}\right)=\sum_{\mathbf{k}, \pm} e^{i \mathbf{k}_{\|} \cdot \mathbf{r}}\left(\begin{array}{ll}
A_{\mathbf{k}, \pm}^{p, n} & e^{ \pm i k_{p} z} \\
B_{\mathbf{k}, \pm}^{h, n} & e^{ \pm i k_{h} z}
\end{array}\right), \\
\left(\begin{array}{c}
u_{\uparrow}^{s} \\
v_{\downarrow}^{s}
\end{array}\right)=\sum_{\mathbf{k}, \pm} e^{i \mathbf{k}_{\|} \cdot \mathbf{r}}\left[\left(\begin{array}{c}
A_{\mathbf{k}, \pm}^{h, s} \\
B_{\mathbf{k}, \pm}^{h, s}
\end{array}\right) e^{ \pm i k^{h} z}+\left(\begin{array}{c}
A_{\mathbf{k}, \pm}^{p, s} \\
B_{\mathbf{k}, \pm}^{p, s}
\end{array}\right) e^{ \pm i k^{p} z}\right] .
\end{gathered}
$$

Here $A$ and $B$ are complex scattering amplitudes and the suband superscripts $n, s, p$, and $h$ denote normal, superfluid, particlelike, and holelike, respectively. We split the vectors $\mathbf{k}$ into its components parallel to the wall $\mathbf{k}_{\|}=\left(k_{x}, k_{y}, 0\right)$ and its $z$ component $k_{p}, k_{h}, k^{p}$, and $k^{h}$ perpendicular to the interface. At fixed energy, there exist eight quasiparticle states, four of which in the $\mathrm{N}$ and four in the SF phase. These states are represented by dots in Fig. 1: filled dots are particlelike states and empty dots are holelike states (the latter have opposite group and phase velocity). By use of the spectra of Eqs. (12) and (13), one relates the wave numbers $k^{p}, k^{h}$, and $k_{h}$ to the wave number $k_{p}$ of a particle in the $\mathrm{N}$ phase,

$$
\begin{gathered}
k_{p}=\left[-k_{\|}^{2}+2 m_{\uparrow}\left(\mu_{\uparrow}-U_{\uparrow}+E_{\alpha}\right) / \hbar^{2}\right]^{1 / 2}, \\
k_{h}=\left\{k_{p}^{2}+2 m_{\uparrow}\left[U_{\uparrow}-\tilde{m} U_{\downarrow}+U_{S}(\tilde{m}-1)-2 \varepsilon_{\alpha}\right] / \hbar^{2}\right\}^{1 / 2}, \\
k^{p, h}=\left[k_{p}^{2}+2 m_{\uparrow}\left(U_{\uparrow}-U_{S}-\chi_{\alpha}^{ \pm}\right) / \hbar^{2}\right]^{1 / 2},
\end{gathered}
$$

where we defined

$$
\begin{gathered}
\varepsilon_{\alpha} \equiv \frac{\left(E_{\alpha}+h_{S}\right)(1+\widetilde{m})}{2}+\frac{\mu_{S}(1-\tilde{m})}{2}, \\
\chi_{\alpha}^{ \pm} \equiv \varepsilon_{\alpha} \mp \sqrt{\varepsilon_{\alpha}^{2}-\widetilde{m} \Delta^{2}},
\end{gathered}
$$

and $\tilde{m} \equiv m_{\downarrow} / m_{\uparrow}$.

\section{B. Scattering diagram}

Let us now use these equations to try to understand the physics of the scattering processes at the interface. Consider for example a particle incident on the interface from the $\mathrm{N}$ side with wave number $k_{p}$ (along the $z$ axis) and energy $E_{\alpha}$; this is represented in Fig. 1 by a curly arrow. Whatever processes occur at the interface, energy must be conserved; but out of the seven remaining states in the $\mathrm{N}$ phase with the same energy, only four have a group velocity directed away from the interface. This implies that $B_{\mathbf{k},-}^{h, n}=A_{\mathbf{k},-}^{p, s}=B_{\mathbf{k},+}^{h, s}=0$. The particle can now undergo either specular reflection to the state marked in Fig. 1 by $-k_{p}$, Andreev reflection to the state $k_{h}$ or it can penetrate the SF as a particlelike (to $k^{p}$ ) or as a holelike quasiparticle (to $-k^{h}$ ). Note that Andreev reflection is also of importance in other ultracold systems such as in fermionic traps without population imbalance [40] and in bosonic traps near a N-SF interface in the absence [41] or presence [42] of a lattice. 


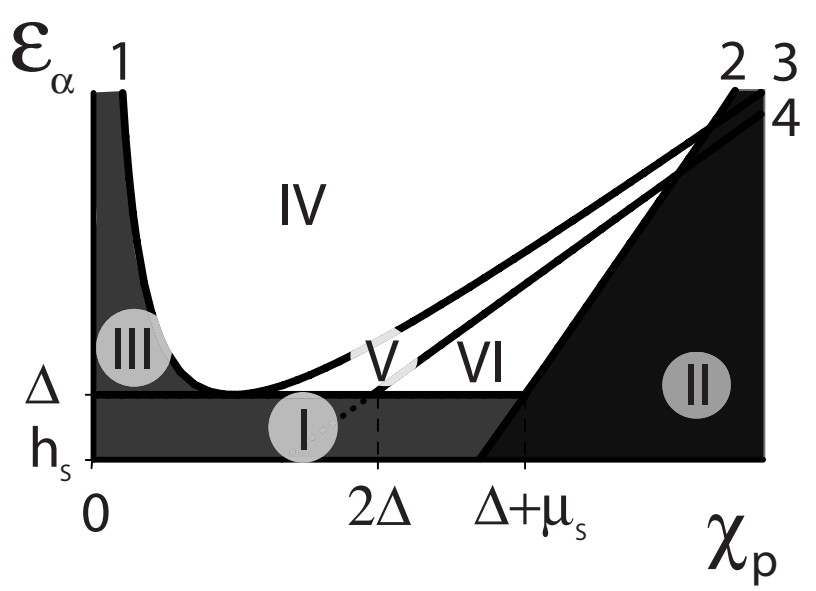

FIG. 2. The various scattering regimes for a particle of species $\uparrow$ which is incident on the interface from the $\mathrm{N}$ side, as a function of its "energy" $\varepsilon_{\alpha}$ [see Eq. (16a)] and $\chi_{p}=\hbar^{2} k_{p}^{2} / 2 m_{\uparrow}$ (where $k_{p}$ is its momentum along the $z$ axis). We assumed here no species mass asymmetry $m_{\uparrow}=m_{\downarrow}$ and no HF potentials $U_{\uparrow, \downarrow, S}=0$; we depict a generic diagram for the more general case $\left(m_{\uparrow} \neq m_{\downarrow}\right.$ and $\left.U_{\uparrow, \downarrow, S} \neq 0\right)$ in Fig. 3. The heavily shaded regimes are energetically forbidden while complete reflection occurs in the lightly shaded regimes. Particles in regime VI may scatter to all states indicated in Fig. 1 by curly arrows. Above line 4 (regimes IV and V), Andreev reflection does not occur, and above curve 3 (regime IV), holelike excitations are also impossible. The functions of the curves 1-4 are given in Eq. (17).

The scattering amplitudes depend on the energy $E_{\alpha}$ of the incident particle, represented by the long-dashed line in Fig. 1. Consider for instance decreasing the energy of the incident particle to a value smaller than the lowest value of the $\alpha$ branch; since no SF states are available, transmission to the $\mathrm{SF}$ is then not possible. Mathematically this is marked by the wave number of one state in the $\alpha$ branch becoming imaginary, indicating the presence of an evanescent wave in the SF. Furthermore, we can also raise the long-dashed line of Fig. 1, that is, increase the energy $E_{\alpha}$, to a value above the local maximum at $k_{z}=0$ of the spectrum of species $\downarrow$ in the $\mathrm{N}$ phase; this will inhibit Andreev reflection.

The crossover of either of the $k_{h}, k^{h}$, and $k^{p}$ and the wave vector $\mathbf{k}_{\|}$from real to imaginary (or vice versa) signifies a change in the scattering mechanism. Defining $\chi_{p}$ $\equiv \hbar^{2} k_{p}^{2} / 2 m_{\uparrow}$, such transitions are encountered when [see Eq. (15)]:

$$
\left\{\begin{array}{r}
1: k^{h}=0 \Rightarrow \chi_{p}=\chi_{\alpha}^{-}-U_{\uparrow}+U_{S}, \\
2: \mathbf{k}_{\|}=0 \Rightarrow \chi_{p}=\mu_{\uparrow}-U_{\uparrow}+E_{\alpha}, \\
3: k^{p}=0 \Rightarrow \chi_{p}=\chi_{\alpha}^{+}-U_{\uparrow}+U_{S}, \\
4: k_{h}=0 \Rightarrow \chi_{p}=2 \varepsilon_{\alpha}-U_{\uparrow}+\tilde{m} U_{\downarrow} \\
-U_{S}(\tilde{m}-1) .
\end{array}\right.
$$

The curves 1-4 separate the possible scattering regimes and are shown in Figs. 2 and 3 where the latter depicts a general (generic) diagram and the former assumes equal species masses and zero HF potentials.

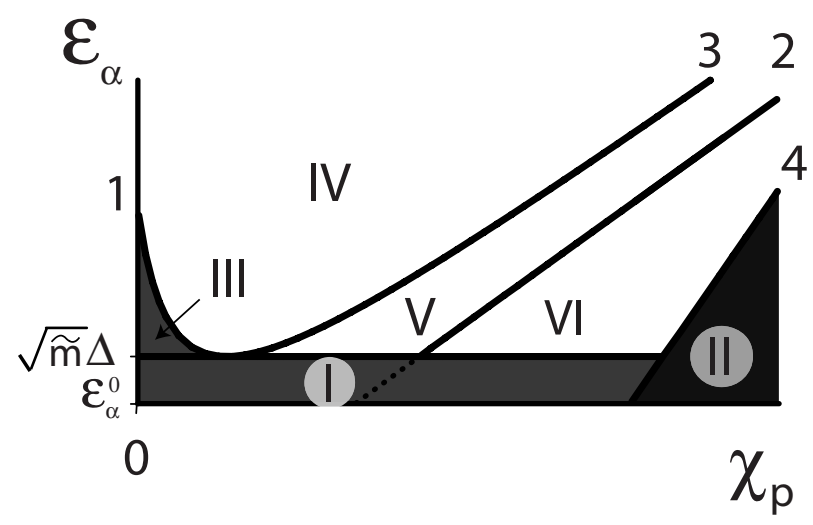

FIG. 3. The same applies as in Fig. 2 except here $m_{\uparrow} \neq m_{\downarrow}$ and $U_{\uparrow, \downarrow, S} \neq 0$. Regime I is bound by threshold line $\varepsilon_{\alpha}=\sqrt{\tilde{m} \Delta}$ and the lower limit $\varepsilon_{\alpha}=\varepsilon_{\alpha}^{0}=\left[h_{S}(1+\tilde{m})+\mu_{S}(1-\tilde{m})\right] / 2$. Compared to Fig. 2, it can be seen that curves $1,2,3$, and 4 are shifted to the right or to the left.

To better understand the various scattering regimes, we switch to working with the energy $\varepsilon_{\alpha}$ of Eq. (16a) instead of $E_{\alpha}$. Recall that $\varepsilon_{\alpha}$ takes values in the interval $\left[\varepsilon_{\alpha}^{0}, \infty\right]$ where $\varepsilon_{\alpha}^{0}=\left[h_{S}(1+\tilde{m})+\mu_{S}(1-\tilde{m})\right] / 2$; clearly $\varepsilon_{\alpha}=E_{\alpha}$ in case $h_{S}=0$ and $m_{\uparrow}=m_{\downarrow}$. If the energy of the particle incident on the interface is small enough or $\varepsilon_{\alpha}<\sqrt{\tilde{m}} \Delta$, there is insufficient energy to excite quasiparticles inside the SF; the particle is then fully reflected as a superposition of a particle and a hole. This regime is labeled I in Figs. 2 and 3. The situation where $E_{\alpha}<\chi_{p}-\mu_{\uparrow}+U_{\uparrow}$, i.e. regime II is physically forbidden since the wave vector of the incident particle (i.e., $\mathbf{k}_{\|}$) is imaginary.

Suppose now that a particle is incoming with energy $\varepsilon_{\alpha}$ above the threshold value $\sqrt{\tilde{m}} \Delta$. Although a particle in regime III appears to have sufficient energy for transmission, in undergoes complete reflection. Particles in regime IV may be transmitted and reflected but all targeted states are particlelike. In both regimes IV and V, there are no reflected holes; that is, Andreev reflection does not occur. However, in regime $\mathrm{V}$, both particlelike and holelike excitations are present in the SF. Finally, in regime VI, both particlelike and holelike excitations, or both Andreev and normal reflection, are allowed.

The blocking of scattering regimes can be understood as being caused by a too large angle of incidence at the interface, similar to total internal reflection. While the critical angle is roughly constant for optical total internal reflection, here the critical angles of incidence depend on the energy of the incoming particle. Generally, a particle of species $\uparrow$ has an angle of incidence $\theta$ which satisfies $\tan \theta=\left|\mathbf{k}_{\|}\right| / k_{p}$, or in terms of the energy,

$$
\tan (\theta)=\left(\frac{\mu_{\uparrow}-U_{\uparrow}+E_{\alpha}}{\chi_{p}}-1\right)^{1 / 2} .
$$

The critical angles for transmission, holelike transmission and Andreev reflection can then be obtained as a function of the energy $E_{\alpha}$ by substitution of $\chi_{p}$ from Eq. (17) into Eq. (18).

To summarize, the lightly shaded regimes in Figs. 2 and 3 describe normal particles of species $\uparrow$ which undergo com- 
plete reflection, while the rightmost, heavily shaded regime is unphysical. Only incident particles in the unshaded regimes may excite quasiparticles inside the SF. If we consider now holes of spin species $\downarrow$ which are incident on the interface from the $\mathrm{N}$ phase, they can only be transmitted in a regime analogous to VI in the diagram. Furthermore, one can find now also the scattering diagram, applied for particles of spin species $\downarrow$ and holes of spin species $\uparrow$ incident on the interface and scattering to the $\beta$ branch. The scattering diagram is then qualitatively similar to that of Fig. 2 and the curves 1-4 are given by Eq. (17) after performing the transformations $h_{S} \rightarrow-h_{S}, \tilde{m} \rightarrow 1 / \tilde{m}, \alpha \rightarrow \beta$, and $\uparrow \leftrightarrows \downarrow$.

\section{Discussion of the scattering diagram}

At this stage, we can discuss the influence of the interaction parameter $k_{F} a$, the HF potentials and the species mass asymmetry on the diagram of Fig. 2 as they may strongly affect the transport properties which will be calculated in Sec. IV.

The interaction regime. First of all, the $\chi_{p}-\varepsilon_{\alpha}$ diagrams of the deep BCS interaction regime and the unitary interaction regime have a different topology. Assume for now that $m_{\uparrow}$ $=m_{\downarrow}$ and that $U_{S}=U_{\uparrow}=U_{\downarrow}=0$. In the deep BCS regime, the relation $2 \Delta \ll \Delta+\mu_{S}$ implies that line 2 in Fig. 2 is shifted horizontally to much higher values of $\chi_{p}$ so that regime VI becomes by far the most important scattering regime. Therefore quasiparticle reflections occur almost only via the Andreev mechanism. It is known that this involves transport of particles, but no transport of energy across the interface. In contrast, at unitarity where $2 \Delta>\Delta+\mu_{S}$ [since $\Delta \approx 1.16 \mu_{S}$ as derived from Eq. (6a) in case $m_{\uparrow}=m_{\downarrow}$ and $\left.U_{\uparrow, \downarrow, S}=0\right]$, phase VI does not even exist. This means that quasiparticles with energy $\varepsilon_{\alpha}$ above the threshold $\Delta$ cannot undergo Andreev reflection but only normal (specular) reflection, by which neither particles nor energy are carried across the interface.

The HF potentials. Assume again that $m_{\uparrow}=m_{\downarrow}$; the HF potentials $U_{S}, U_{\uparrow}$, and $U_{\downarrow}$ are seen to be present in expression (17) which describe the curves 1-4 of Fig. 2. However, it follows from these equations that the HF potentials induce only horizontal shifts of the curves 1-4 but do not affect the upper and the lower bounds of regime I. Whereas line 2 is shifted to the right, the curves 1,3 , and 4 are always shifted to the left. This can be deduced from the fact that $U_{S}<U_{\uparrow}$ $\leq 0$ and $U_{\downarrow}<U_{\uparrow} \leq 0$ since the interactions are attractive and the density of the SF exceeds the density of the $\mathrm{N}$ phase.

The species mass asymmetry. In the presence of a species mass asymmetry $m_{\uparrow} \neq m_{\downarrow}$, regime I may become either larger or smaller. This is due to a shift of its lower bound $\varepsilon_{\alpha}^{0}$ $=\left[h_{S}(1+\tilde{m})+\mu_{S}(1-\tilde{m})\right] / 2$ [obtained by taking $E_{\alpha}=0$ in Eq. (16a) $]$ and a shift of the upper bound $\varepsilon_{\alpha}=\sqrt{\tilde{m} \Delta}$. It is seen that both shifts have the same sign but can either increase or decrease the surface area of regime I. Also, the species mass asymmetry induces a shift of line 4 as is seen from Eq. (17).

\section{Transmission coefficients}

In order to study the transport properties in Sec. IV, we first seek to relate the scattering amplitudes $A$ and $B$ of Eq. (14) to the transmission coefficients. The amplitudes are de-

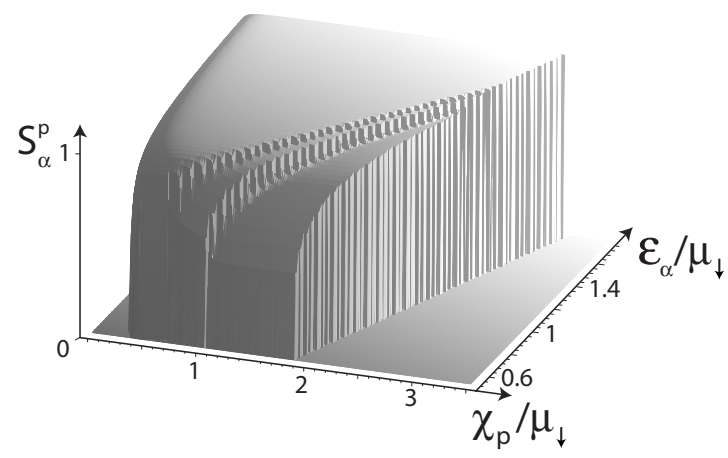

FIG. 4. The transmission coefficient $S_{\alpha}^{p}$ for $\uparrow$ particles scattering to the $\alpha$ channel as a function of the energy $\varepsilon_{\alpha}$ [see Eq. (16a)] and the variable $\chi_{p} \equiv \hbar^{2} k_{p}^{2} /\left(2 m_{\uparrow}\right)$, both in units of $\mu_{\downarrow}$, in the case of $m_{\uparrow}=m_{\downarrow}$ and with the HF potentials set to zero. One can distinguish the regimes IV, V, and VI (see diagram of Fig. 2) of nonzero $S_{\alpha}^{p}$. We choose the value $\Delta=0.5 \mu$ and used the coexistence condition $h_{S}$ $=\Delta / \sqrt{2}$. Regime I where $\varepsilon_{\alpha}<\Delta$ is not depicted in this figure since there $S_{\alpha}^{p}=0$. For values slightly above the threshold $\varepsilon_{\alpha}=\Delta$, one observes the square-root behavior of relation (20). We give the analytical expressions for $S_{\alpha}^{p}$ in Eq. (A5) in the Appendix.

termined by matching the quasiparticle wave functions of Eq. (14) and their derivatives at the interface $z=0$ [43]. In its most general form, this leads to the equations,

$$
\begin{aligned}
& \left(\begin{array}{c}
A_{\mathbf{k},+}^{p, n}+A_{\mathbf{k},-}^{p, n} \\
B_{\mathbf{k},+}^{h, n}+B_{\mathbf{k},-}^{h, n} \\
k_{p}\left(A_{\mathbf{k},+}^{p, n}-A_{\mathbf{k},-}^{p, n}\right) \\
k_{h}\left(B_{\mathbf{k},+}^{h, n}-B_{\mathbf{k},-}^{h, n}\right)
\end{array}\right) \\
& =\left(\begin{array}{c}
A_{\mathbf{k},+}^{p, s}+A_{\mathbf{k},+}^{h, s}+A_{\mathbf{k},-}^{p, s}+A_{\mathbf{k},-}^{h, s} \\
\boldsymbol{s}_{p} B_{\mathbf{k},+}^{p, s}+\boldsymbol{s}_{h} B_{\mathbf{k},+}^{h, s}+\boldsymbol{s}_{p} B_{\mathbf{k},-}^{p, s}+\boldsymbol{s}_{h} B_{\mathbf{k},-}^{h, s} \\
k^{p}\left(A_{\mathbf{k},+}^{p, s}-A_{\mathbf{k},-}^{p, s}\right)+k^{h}\left(A_{\mathbf{k},+}^{h, s}-A_{\mathbf{k},-}^{h, s}\right) \\
k^{p} \mathbf{s}_{p}\left(B_{\mathbf{k},+}^{p, s}-B_{\mathbf{k},-}^{p, s}\right)+k^{h} \mathbf{s}_{h}\left(B_{\mathbf{k},+}^{h, s}-B_{\mathbf{k},-}^{h, s}\right)
\end{array}\right),
\end{aligned}
$$

where we defined $\varsigma_{p, h} \equiv \chi_{\alpha}^{ \pm} / \Delta$. As argued before, depending on the various scattering regimes, one must set some scattering amplitudes to zero. One finds

$$
\begin{cases}\text { for regime VI: } & B_{\mathbf{k},-}^{h, n}=A_{\mathbf{k},-}^{p, s}=B_{\mathbf{k},+}^{h, s}=0, \\ \text { for regime V: } & B_{\mathbf{k},+}^{h, n}=A_{\mathbf{k},-}^{p, s}=B_{\mathbf{k},+}^{h, s}=0, \\ \text { for regime IV \& V: } & B_{\mathbf{k},+}^{h, n}=A_{\mathbf{k},-}^{p, s}=B_{\mathbf{k},-}^{h, s}=0, \\ \text { for holes: } & A_{\mathbf{k},+}^{p, n}=A_{\mathbf{k},-}^{p, s}=B_{\mathbf{k},+}^{h, s}=0 .\end{cases}
$$

We give the solutions for the scattering amplitudes in expressions (A1)-(A4) in the Appendix.

From the HFBdG Eq. (2) it readily follows that the quasiparticle density $\rho_{\alpha}(\mathbf{r})=\left|u_{\uparrow}(\mathbf{r})\right|^{2}+\left|v_{\downarrow}(\mathbf{r})\right|^{2}$ and the quasiparticle currents,

$$
\mathbf{j}_{\alpha}=-\frac{i}{2 m_{\uparrow}}\left[u_{\uparrow}^{*} \boldsymbol{\nabla} u_{\uparrow}-u_{\uparrow} \boldsymbol{\nabla} u_{\uparrow}^{*}\right]-\frac{i}{2 m_{\downarrow}}\left[v_{\downarrow} \boldsymbol{\nabla} v_{\downarrow}^{*}-v_{\downarrow}^{*} \boldsymbol{\nabla} v_{\downarrow}\right],
$$

satisfy the "continuity equation" $\partial \rho_{\alpha} / \partial t+\nabla \cdot \mathbf{j}_{\alpha}=0$. Note, however, that they do not express conservation of particles or mass. The same expressions are valid for the $\beta$ branch after the transformation $\uparrow \rightleftarrows \downarrow$. We define now the transmission 
coefficient $S_{\alpha}^{p}\left(\varepsilon_{\alpha}, \chi_{p}\right)$ of an incident particle of energy $\varepsilon_{\alpha}$ and momentum $\mathbf{k}$ as the ratio of the transmitted to the incoming current along the $z$ axis. As argued before, the transmission coefficients vanish in regimes I, II, and III of Fig. 2; for regimes IV, V, and VI (and for holes) we give their analytical forms in expression (A5) in the Appendix. Figure 4 shows the transmission coefficients as a function of $\varepsilon_{\alpha}$ and $\chi_{p}$ in case of $m_{\uparrow}=m_{\downarrow}$ and $U_{\uparrow, \downarrow, S}=0$. One clearly recognizes the three regimes (IV, V, and VI) of the diagram in Fig. 2 with nonzero transmission coefficients. For energies $\varepsilon_{\alpha}$ slightly above the transmission threshold $\varepsilon_{\alpha} \approx \sqrt{\tilde{m}} \Delta$ (for regimes $\mathrm{V}$ and VI) one can analytically find the following behavior:

$$
S_{\alpha}^{p}\left(\varepsilon_{\alpha}, \chi_{p}\right) \propto \sqrt{\varepsilon_{\alpha}-\sqrt{\tilde{m}} \Delta} .
$$

This is similar to the case of particles scattering from a HF potential of height $\sqrt{\widetilde{m}} \Delta$ [44]. In Fig. 4, one indeed observes that $S_{\alpha}^{p}$ grows fast for energies close to the gap value $\varepsilon_{\alpha}$ $=\sqrt{\tilde{m}} \Delta$ (which, in Fig. 4 is taken to be $0.5 \mu_{\downarrow}$ ). Moreover, in the BCS regime wherein, by the Andreev approximation, one can assume $k^{p}=k^{h}=k_{p}=k_{h}$, the transmission coefficients reduce to

$$
S_{\alpha}^{p, h}=\frac{2 \sqrt{\varepsilon_{\alpha}^{2}-\Delta^{2} \tilde{m}}}{\varepsilon_{\alpha}+\sqrt{\varepsilon_{\alpha}^{2}-\Delta^{2} \tilde{m}}} .
$$

Apart from the species mass asymmetry $\tilde{m}$, this is the expression which was obtained by Andreev in Ref. [23].

\section{HEAT TRANSPORT THROUGH THE INTERFACE}

Thermally excited particles incident on the surface will be reflected unless they have an energy of at least $2(\sqrt{\tilde{m}} \Delta$ $\left.-\varepsilon_{\alpha}^{0}\right) /(\tilde{m}+1)$. At low enough temperatures, not many particles will have enough energy to penetrate. This leads to a significant decrease in heat conductivity through the interface, a phenomenon well known for superconductors $[23,45]$. We will now argue that the same blocking of energy transport occurs for imbalanced fermion gases, and discuss the influence of the interaction regime on this phenomenon.

Heat transport across the interface can be studied using the transmission coefficients calculated in Sec. III D. Using the relation,

$$
\int \frac{d^{3} \mathbf{k}}{(2 \pi)^{3}}=\frac{m_{\uparrow}}{2 \pi^{2}(1+\tilde{m}) \hbar^{2}} \int d \varepsilon_{\alpha} \int d k_{p},
$$

one can write the particlelike heat flux through the interface and to the $\alpha$ branch as

$$
\begin{aligned}
W_{\alpha}^{p}= & \frac{m_{\uparrow}}{\pi^{2} \hbar^{3}(\tilde{m}+1)^{2}} \int d \varepsilon_{\alpha} \int d \chi_{\alpha} \\
& \times\left(\varepsilon_{\alpha}-\varepsilon_{\alpha}^{0}\right) f\left[E_{\alpha}\left(\varepsilon_{\alpha}\right)\right] S_{\alpha}^{p}\left(\varepsilon_{\alpha}, \chi_{\alpha}\right),
\end{aligned}
$$

where $f$ is the Fermi-Dirac distribution and the integration is performed over all regimes of the $\chi_{p}-\varepsilon_{\alpha}$ plane. For the heat flux $W_{\alpha}^{h}$ carried by hole excitations, the integration is only over regime VI and by use of $d k_{h}^{2}=d k_{p}^{2}$, we can obtain the same expression as Eq. (21) but with $m_{\downarrow}$ instead of $m_{\uparrow}$ and with $S_{\alpha}^{h}$ instead of $S_{\alpha}^{p}$, as given in Eq. (A8). By the same approach, the contributions of the $\beta$ branch are then found by performing the transformations $h_{S} \rightarrow-h_{S}, \tilde{m} \rightarrow 1 / \tilde{m}, \alpha \rightarrow \beta$, and $\uparrow \leftrightarrows \downarrow$.

In equilibrium, quasiparticles are incident on both sides of the interface such that the N-SF flux is balanced by an equal SF-N flux. Suppose, however, that the temperature on the $\mathrm{N}$ side is $\delta T$ higher than on the SF side (and $\delta T / T \ll 1$ ). A net heat flow $\delta Q=\delta Q^{\alpha}+\delta Q^{\beta}$ then results from the scattering to the $\alpha$ channel $\left(\delta Q^{\alpha}\right)$ and to the $\beta$ channel $\left(\delta Q^{\beta}\right)$. For small temperature differences, we may write $\delta Q^{\alpha}=\kappa^{\alpha} \delta T$ with $\kappa^{\alpha}$ the heat conductivity caused by scattering to the $\alpha$ channel. Within the Kapitza approach, we have

$$
\kappa^{\alpha}=\frac{\partial\left(W_{\alpha}^{p}+W_{\alpha}^{h}\right)}{\partial\left(k_{B} T\right)} .
$$

In the deep BCS regime, where the Andreev approximation may be used, one may obtain an analytical expression for $\kappa$. Taking $k^{p}=k^{h}=k_{p}=k_{h}$ and assuming the temperature to satisfy $k_{B} T \ll \Delta \ll \mu_{S}$ effects regime VI of Figs. 2 and 3 to be the sole important scattering regime; in other words, all reflections are due to Andreev reflection. We obtain for the heat flux to the $\alpha$ channel [see Eq. (21)],

$$
\begin{aligned}
W_{\alpha}^{p}+W_{\alpha}^{h}= & \frac{\sqrt{\pi}\left(m_{\uparrow}+m_{\downarrow}\right) \mu_{\uparrow} e^{-2 \beta\left(\Delta \sqrt{\tilde{m}}-\varepsilon_{\alpha}^{0}\right) /(\tilde{m}+1)}}{2 \pi^{2} \hbar^{3} \sqrt{\tilde{m}+1}} \\
& \times \frac{\left[\Delta \sqrt{\tilde{m}}-\varepsilon_{\alpha}^{0}+3 k_{B} T(\tilde{m}+1) / 4\right]\left(k_{B} T\right)^{3 / 2}}{\sqrt{\Delta \sqrt{\tilde{m}}}} .
\end{aligned}
$$

For the $\alpha$ channel the heat conductivity of Eq. (22) in the $\mathrm{BCS}$ regime is then obtained,

$$
\begin{aligned}
\kappa_{B C S}^{\alpha}= & \frac{\sqrt{\pi}\left(m_{\uparrow}+m_{\downarrow}\right) e^{-2 \beta\left(\Delta \sqrt{\tilde{m}}-\varepsilon_{\alpha}^{0}\right) /(\tilde{m}+1)}}{\pi^{2} \hbar^{3}(\tilde{m}+1)^{3 / 2}} \\
& \times \frac{\left(\Delta \sqrt{\tilde{m}}-\varepsilon_{\alpha}^{0}\right)\left[\Delta \sqrt{\tilde{m}}-\varepsilon_{\alpha}^{0}+3 k_{B} T(\tilde{m}+1) / 2\right]}{\sqrt{k_{B} T \Delta \sqrt{\tilde{m}}}},
\end{aligned}
$$

which amounts to Andreev's result when $h_{S}=0$ and $m_{\uparrow}=m$ [23]. The heat flux and heat conductivity of the $\beta$ channel is again found after the transformation $\tilde{m} \rightarrow 1 / \tilde{m}, \uparrow \rightleftarrows \downarrow, h_{S} \rightarrow$ $-h_{S}$, and $\alpha \rightarrow \beta$. The energy carried by the $\beta$ branch is a factor $e^{-4 \beta h_{S} /(\tilde{m}+1)}$ lower than that of the $\alpha$ branch and due to coexistence $h \sim \Delta$ and since $\Delta \gg T$, it can be neglected.

Equation (23) demonstrates that $\kappa$ decays exponentially fast with decreasing temperature. This can be understood as follows. For low enough temperature, i.e., $k_{B} T \ll \sqrt{\tilde{m}} \Delta$, only incident particles (and holes) with energies slightly above the threshold $\varepsilon_{\alpha} \approx \sqrt{m} \Delta$ can contribute to the heat conductivity; they have, however, a very low statistical weight,

$$
f \propto e^{-2 \beta\left(\sqrt{\tilde{m}} \Delta-\varepsilon_{\alpha}^{0}\right) /(\widetilde{m}+1)} \ll 1 .
$$

It is this weight factor which also appears in expression (23). The question now is: what will happen to this exponential decay if we tune the interactions from the BCS to the unitary regime? In order to answer this, we have numerically calculated the ratio of $\kappa \equiv \kappa^{\alpha}+\kappa^{\beta}$ to the conductivity in the $\mathrm{N}$ 


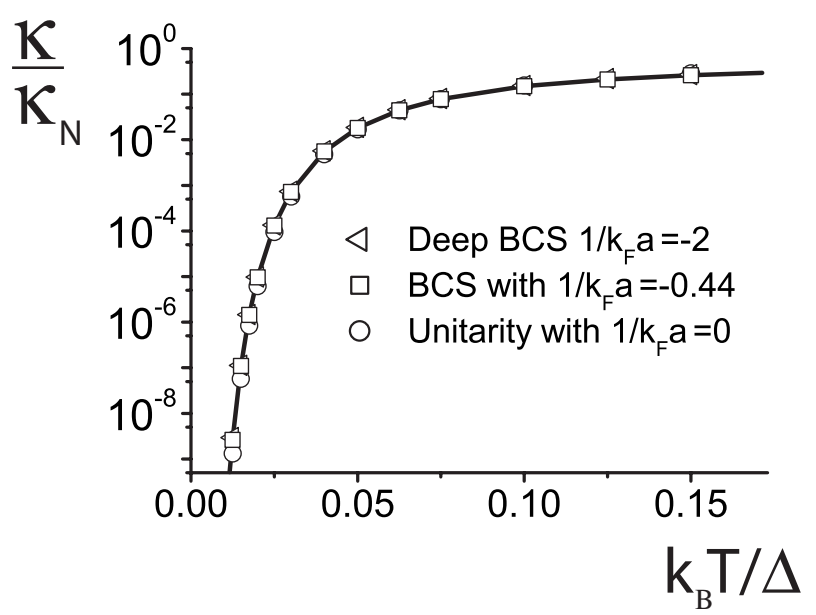

FIG. 5. The thermal conductivity $\kappa$ across the N-SF interface divided by the normal-phase conductivity $\kappa_{N}$ against $k_{B} T / \Delta$ for the unitary, BCS and deep BCS regimes when $m_{\uparrow}=m_{\downarrow}$ and $U_{\uparrow, \downarrow, S}=0$. For $k_{B} T \leqq 0.1 \Delta, \kappa / \kappa_{N}$ drops dramatically (notice the logarithmic scale). The curve represents the analytical result obtained using of the Andreev approximation, Eq. (23). At unitarity, $\Delta=0.69 k_{B} T_{F}$, in the BCS case $\Delta=0.25 k_{B} T_{F}$ and in the deep BCS case $\Delta$ $=0.002 k_{B} T_{F}$. For large values of the temperature, one finds that $\kappa / \kappa_{N}=1$.

phase $\kappa_{N}=k_{B} T\left(\mu_{\uparrow} m_{\uparrow}+\mu_{\downarrow} m_{\downarrow}\right) / \pi^{2} \hbar^{3}$, and show this in Fig. 5 as a function of $k_{B} T / \Delta$ and we assumed $m_{\uparrow}=m_{\downarrow}$ and $U_{\uparrow, \downarrow}$ $=0$. We find that $\kappa / \kappa_{N}$ decreases drastically below $k_{B} T$ $\approx 0.1 \Delta$ and is independent of the interaction parameter $k_{F} a$. Also in Fig. 5 we plot the heat conductivity of Eq. (23); it is seen to give a good fit for all scattering regimes [46]. The reason for this good agreement is that the threshold line $\varepsilon_{\alpha}$ $=\sqrt{m} \Delta$ of the diagram of Fig. 2 is unaffected by changing the scattering regime. Furthermore, relation (20) which describes the transmission coefficient slightly above the threshold line guarantees that $S_{\alpha}^{p}$ displays the same behavior in regimes $\mathrm{V}$ and VI.

As argued before, the main effect of varying $1 /\left(k_{F} a\right)$ from large and negative (deep BCS) to zero (unitarity), is to shift the curves 1-4 in diagram of Fig. 2. Both the strong exponential decay in $\sqrt{\tilde{m}} \Delta /\left(k_{B} T\right)$ and the square-root dependence of $S_{\alpha}^{p}$ on $\varepsilon_{\alpha}-\sqrt{\tilde{m}} \Delta$ are unaffected by the variation of $1 /\left(k_{F} a\right)$ and the introduction of the Hartree potentials, hence the very similar behavior of $\kappa / \kappa_{N}$ for all scattering regimes under study. On the other hand, what is different between the scattering regimes is the value of $\Delta$; it is small in the sense that $\Delta \ll k_{B} T_{F}$ in the BCS limit and is comparable with $k_{B} T_{F}$ at unitarity.

\section{EQUILIBRATION TIMESCALES}

The introduction of the Hartree potentials has two implications on the heat resistivity. First of all, the scattering diagram will change by horizontal shifts of lines 1-4; as argued before, this does not affect the steep exponential decay of resistivity. Secondly, the coexistence condition will change, that is, the relation between $\Delta$ and $h$ will change. This, on the other hand may strongly affect the exponential decay as is

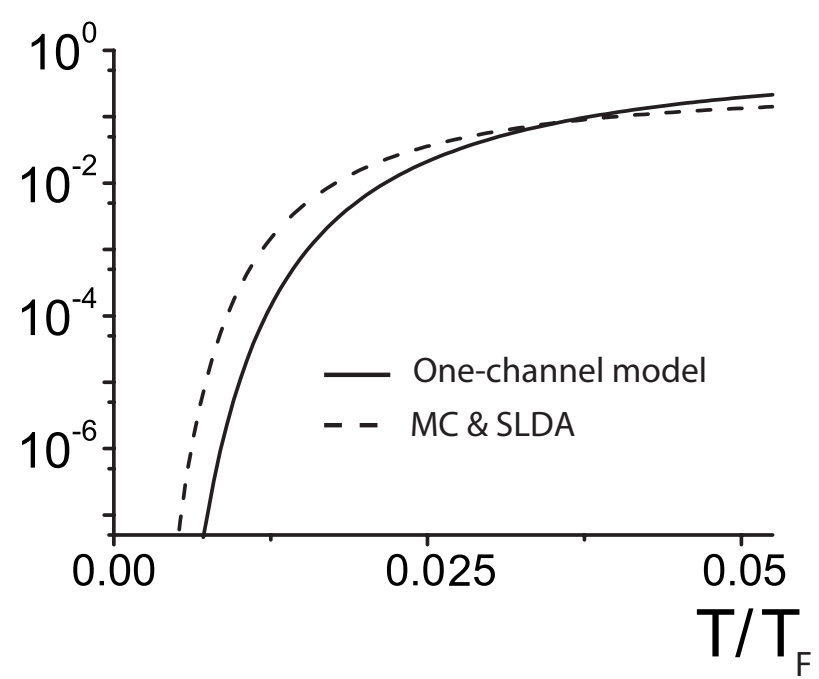

FIG. 6. The thermal conductivity $\kappa$ across the N-SF interface divided by the normal-phase conductivity $\kappa_{N}$ against $T / T_{F}$ at the interface in the unitary regime. The full line depicts the Andreev approximation wherein the coexistence condition is taken from the one-channel model. The dotted line is the Andreev approximation with the coexistence condition is taken from Monte Carlo simulations [22] and a superfluid local-density approximation [33]. At the lowest experimental values, that is when $T<0.03 T_{F}$, the conductivity is already very low.

seen from Eq. (24). At unitarity, the relation between $\Delta$ and $h$ is known from Monte Carlo [22] simulations and a superfluid local-density approximation (SLDA) [33]. From these we adapt the values $\Delta=0.504 k_{B} T_{F}, \quad U_{S}=-0.516 k_{B} T_{F}, \mu_{S}$ $=0.936 k_{B} T_{F}$ [33], and finally $h_{S}=0.48\left(\mu_{\uparrow}+\mu_{\downarrow}\right)$ [22]. In Fig. 6 , we compare the Andreev approximation with values of the one-channel model (full line) and of the MC and SLDA results (dashed line). We see that the one-channel model predicts the sharp decrease in $\kappa$ to be at higher temperatures than the MC and SLDA results.

We find that the interface conductivity at the lowest experimental temperatures is very low in the sense that the resulting characteristic equilibration time is of order of seconds, i.e., the trap lifetime.

We explain now how we estimate the characteristic equilibration time $\tau$. Consider again a temperature bias $\delta T$ across the interface. We are interested in the resulting total heat flux $\delta W$, induced by the excess heat $\Sigma_{i=\uparrow, \downarrow} \delta Q_{i}$ in the $\mathrm{N}$ phase. By definition, the heat flux $W$ is the amount of heat transferred per unit time per unit surface area. One can therefore estimate $\tau$ by the relation $\delta W=\Sigma_{i=\uparrow, \downarrow} \delta Q_{i} /(\tau \mathcal{A})$ with $\mathcal{A}$ the interface area. Moreover, at position $\mathbf{r}$ in the degenerate $\mathrm{N}$ phase $i=\uparrow, \downarrow$, one has $\delta Q_{i}(\mathbf{r})=\pi^{2} \rho_{i}(\mathbf{r}) T \delta T d \mathbf{r} /\left[2 \mu_{i}(\mathbf{r})\right]$ with $\rho_{i}$ the particle density and $i=\uparrow, \downarrow[47]$. Combining this with $\delta W$ $=\kappa k_{B} \delta T$, one finds

$$
\tau=\frac{\pi^{2} k_{B} T}{2 \mathcal{A} \kappa} \sum_{i=\uparrow, \downarrow} \int_{N} \frac{\rho_{i}(\mathbf{r})}{\mu_{i}(\mathbf{r})} d \mathbf{r} .
$$

Assume now an isotropic trap of $10^{7}$ particles at polarization 0.5 with radial trapping frequency $458 \mathrm{~Hz}$ such that the local-density approximation is valid [5]. For this configura- 
tion, we calculate that the characteristic time of relaxation $\tau$ is of order seconds when $T / T_{F} \lesssim 0.02$. This means that according to our estimates, the relaxation time is of the order of the lifetime of the system at the lowest attainable experimental temperature $T / T_{F}=0.03$ such that in these experiments, the $\mathrm{N}$ and $\mathrm{SF}$ phases may not be equilibrated.

At least two interesting experimental consequences of the low thermal conductivity for ultracold gases are different to what is the case for a SC. First of all, in a superconductor, the heat conductivity has a lattice component which dominates the electron component at low temperatures; the lack of such a component in the system under study makes the decrease in the conductivity more significant [48]. Secondly, at very low temperatures, a N-SF temperature gradient will entail a pressure difference $\delta P$ across the interface, and therefore the physical movement of the interface; this is impossible for normal-superconductor junctions. Taking $\delta P$ $=(\partial P / \partial T) \delta T$ and an infinite thermal resistivity, one finds [47]

$$
\delta P=\frac{5 \pi^{2}}{6} \sum_{i=\uparrow, \downarrow} P_{i 0} \frac{k_{B} T}{\mu_{i}} \delta\left(\frac{k_{B} T}{\mu_{i}}\right),
$$

where $P_{i 0}$ is the $T=0$ pressure of species $i=\uparrow, \downarrow$. Depending on the time scales involved, mechanical equilibrium might be reached before thermal equilibrium.

\section{DISCUSSION}

The step-function model for the gap [see Eq. (11)] is adequate for tackling the problem at hand, that is for estimating the heat conductivity across the interface.

First of all, this model should give qualitatively correct results as long as the quasiparticle wavelengths are of the same order or less as the length over which the gap varies. Indeed, this is clearly valid in the BCS limit, when the healing length for the gap, further denoted as $\xi$, is of order $\xi_{0}$ $=\hbar^{2} k_{F} / m_{+} \Delta$ and $\Delta \ll \hbar^{2} k_{F}^{2} / m_{+}$. Moreover, near unitarity, the step-function model is also justified since the length of variation in the gap is smaller than the quasiparticle wavelength [20]. Therefore, the step function of Eq. (11) serves as a good approximation for all regimes under consideration.

Second, the step model for the gap is justified whenever the interface width must be small as compared to the variation length of the trapping potential. This is also realized in experiments.

We argue now that, even if we would take into account the exact interface profile, our results would hardly be affected. By solving the Bogoliubov-de Gennes equations in a self-consistent manner, the interface profile for the gap could be determined $[49,50]$. At zero temperature, the concomitant interface width is of order $\xi_{0}=\hbar^{2} k_{F} / m_{+} \Delta$. As was shown in Ref. [51], such smooth interface gives rise to a zero transmission coefficients for particles with energy below the gap, and above the gap, smaller transmission coefficients than predicted by our step-function model. This simply means that particles incoming to the interface with energy below the gap cannot cross over to the superfluid phase and therefore they cannot carry energy across the interface. Hence our conclusions of a blocking of energy transport across the inter- face remain valid, independent of the interface width. Note also that, due to the decreased transmission coefficient above the gap, the heat conductivity will be even smaller than predicted here.

The same conclusion can be drawn at finite temperatures: particles with energy below the gap cannot penetrate the superfluid and the conclusions of Ref. [51] concerning the transmission coefficients will still be valid. Indeed, since it is known that the interface width increases with temperature as it varies between $\xi(T=0)=\xi_{0}$ and $\xi(T) \propto \xi_{0}\left(1-T / T_{c}\right)^{-1 / 2}$ near $T=T_{c}$ [25], $\xi$ is certainly of the order of $\xi_{0}$ at the experimentally relevant temperatures, that is below $0.1 T_{c}$. This can be seen in Refs. [52,53] where the self-consistent calculations were performed for various temperatures and even for the strong-coupling regime [52].

In our model, we do not take into account the temperature dependence of the gap. However, this is well justified as the gap $\Delta(T)$ depends very weakly on temperature at low $T$. In the BCS limit it is well known that $\Delta(T)$ goes as $\Delta(T)$ $-\Delta(T=0) \propto-\sqrt{T} \exp (-\Delta(0) / T$ ) (see for example in Ref. [54]). Moreover, this weak variation is also present in the strong-coupling limit [55] and can be understood within the single-channel model from the appearance of the factor $\exp (-\Delta / T)$ in the finite-temperature gap equation.

\section{CONCLUSION}

We have studied the effects of a N-SF interface in experiments on trapped imbalanced fermion gases. We clarify the nature of the possible processes that may occur when a particle is incident onto the interface. Such particles will be thermally excited at any finite temperature, and act as carriers of energy, thus eventually causing thermal equilibration between the $\mathrm{N}$ and SF phases.

We find that reflection (both Andreev and normal, or specular) of low-energy particles off the interface in experiments on trapped imbalanced fermion gases causes a suppression of energy transport from one phase to the other. At low enough temperatures (comparable to the currently accessible temperatures in experiments), this suppression grows exponentially with decreasing temperature. This, in turn, delays thermal equilibration of the system, which may result in a temperature difference between the $\mathrm{N}$ and the SF. Our estimates of the timescales for this equilibration are in the seconds, which indicates that this effect is experimentally relevant.

The incorporation of this temperature difference may allow current models to finally resolve the difficulties in explaining the experiments.

\section{ACKNOWLEDGMENTS}

It is a pleasure to thank Henk Stoof and Koos Gubbels for discussions and useful suggestions. We acknowledge partial support by Project No. FWO G.0115.06; A.L. is supported by Project No. GOA/2004/02 and B.V.S. by the Research Fund K.U.Leuven. 


\section{APPENDIX}

Assume particles of species $\uparrow$ incident on the interface with momentum $k_{p}$ and energy $\varepsilon_{\alpha}$. Define first $\varsigma_{p, h} \equiv \chi_{\alpha}^{ \pm} / \Delta$ and

$$
\begin{gathered}
\mathcal{J}_{0} \equiv \varsigma_{p}\left(k^{h}-k_{p}\right)\left(k^{p}-k_{h}\right)-\varsigma_{h}\left(k^{h}+k_{h}\right)\left(k^{p}+k_{p}\right), \\
\mathcal{J}_{1} \equiv \varsigma_{p}\left(k^{h}-k_{p}\right)\left(k^{p}+k_{h}\right)-\varsigma_{h}\left(k^{h}-k_{h}\right)\left(k^{p}+k_{p}\right), \\
\mathcal{J}_{2} \equiv \varsigma_{p}\left(-k^{h}-k_{p}\right)\left(k^{p}+k_{h}\right)-\varsigma_{h}\left(-k^{h}-k_{h}\right)\left(k^{p}+k_{p}\right) .
\end{gathered}
$$

If the coordinate $\left(\chi_{p}, \varepsilon_{\alpha}\right)$ with $\chi_{p} \equiv \hbar^{2} k_{p}^{2} /\left(2 m_{\uparrow}\right)$ is positioned in regime VI of Fig. 2, the matching of the wave functions and their derivatives at $z=0$ leads to the coefficients,

$$
\left(\begin{array}{c}
A_{\mathbf{k},-}^{p, n} \\
B_{\mathbf{k},+}^{h, n} \\
A_{\mathbf{k},+}^{p, s} \\
B_{\mathbf{k},-}^{h, s}
\end{array}\right)=\frac{A_{\mathbf{k},+}^{p, n}}{\mathcal{J}_{0}}\left(\begin{array}{c}
\varsigma_{p}\left(k^{h}+k_{p}\right)\left(k_{h}-k^{p}\right) \\
-\varsigma_{h}\left(k^{h}+k_{h}\right)\left(k_{p}-k^{p}\right) \\
-2 \tilde{m} k_{p}\left(k^{h}+k^{p}\right) \\
-2 \varsigma_{h} k_{p}\left(k^{h}+k_{h}\right) \\
-2 \varsigma_{p} k_{p}\left(k^{p}-k_{h}\right)
\end{array}\right) .
$$

For regime V (see Fig. 2), we get

$$
\left(\begin{array}{c}
A_{\mathbf{k},-}^{p, n} \\
B_{\mathbf{k},-}^{h, n} \\
A_{\mathbf{k},+}^{p, s} \\
B_{\mathbf{k},-}^{h, s}
\end{array}\right)=\frac{A_{\mathbf{k},+}^{p, n}}{\mathcal{J}_{1}}\left(\begin{array}{c}
\varsigma_{p}\left(k^{h}+k_{p}\right)\left(-k^{p}-k_{h}\right) \\
-\varsigma_{h}\left(k^{h}-k_{h}\right)\left(k_{p}-k^{p}\right) \\
-2 \tilde{m} k_{p}\left(k^{h}+k^{p}\right) \\
-2 \varsigma_{h} k_{p}\left(k^{h}-k_{h}\right) \\
-2 \varsigma_{p} k_{p}\left(k^{p}+k_{h}\right)
\end{array}\right) .
$$

For regime IV (see Fig. 2), we get

$$
\left(\begin{array}{c}
A_{\mathbf{k},-}^{p, n} \\
B_{\mathbf{k},-}^{h, n} \\
A_{\mathbf{k},+}^{p, s} \\
B_{\mathbf{k},+}^{h, s}
\end{array}\right)=\frac{A_{\mathbf{k},+}^{p, n}}{\mathcal{J}_{2}}\left(\begin{array}{c}
\mathrm{s}_{p}\left(-k^{h}+k_{p}\right)\left(-k^{p}-k_{h}\right) \\
-\varsigma_{h}\left(-k^{h}-k_{h}\right)\left(k_{p}-k^{p}\right) \\
-2 \tilde{m} k_{p}\left(-k^{h}+k^{p}\right) \\
-2 s_{h} k_{p}\left(-k^{h}-k_{h}\right) \\
-2 s_{p} k_{p}\left(k^{p}+k_{h}\right)
\end{array}\right) .
$$

For holes of species $\downarrow$ which are incident on the interface, and which necessarily must have energy and momentum in regime VI, the scattering amplitudes are

$$
\left(\begin{array}{c}
B_{\mathbf{k},+}^{h, n} \\
A_{\mathbf{k},-}^{p, n} \\
A_{\mathbf{k},+}^{p, s} \\
B_{\mathbf{k},-}^{h, s}
\end{array}\right)=\frac{B_{\mathbf{k},-}^{h, n}}{\mathcal{J}_{0}}\left(\begin{array}{c}
\varsigma_{h}\left(k^{h}-k_{h}\right)\left(k^{p}+k_{p}\right) \\
-\varsigma_{p}\left(k^{h}-k_{p}\right)\left(k^{p}+k_{h}\right) \\
-2 k_{h}\left(k^{h}+k^{p}\right) \\
-2 k_{h}\left(k^{h}-k_{p}\right) \\
-2 k_{h}\left(k^{p}+k_{p}\right)
\end{array}\right) .
$$

The transmission coefficients for the different regimes are

For regime VI: $S_{\alpha}^{p}=\left[4 k^{p} k_{p}\left(k^{h}+k_{h}\right)^{2}\left(s_{h}^{2}-\tilde{m}\right)+4 k^{h} k_{p}\left(k_{h}\right.\right.$

$$
\left.\left.-k^{p}\right)^{2}\left(\tilde{m}-s_{p}^{2}\right)\right] / \mathcal{J}_{0}^{2} \text {. }
$$

(A5a)

For regime V: $S_{\alpha}^{p}=\left[4 k^{p} k_{p}\left|k^{h}-k_{h}\right|^{2}\left(s_{h}^{2}-\tilde{m}\right)+4 k^{h} k_{p} \mid k_{h}\right.$

$$
\left.+\left.k^{p}\right|^{2}\left(\tilde{m}-\varsigma_{p}^{2}\right)\right] /\left|\mathcal{J}_{1}\right|^{2} \text {. }
$$

$$
\text { For regime IV: } S_{\alpha}^{p}=\frac{4 k^{p} k_{p}\left|k^{h}+k_{h}\right|^{2}\left(s_{h}^{2}-\tilde{m}\right)}{\left|\mathcal{J}_{2}\right|^{2}} \text {. }
$$

For holes: $S_{\alpha}^{h}=\left[4 k^{p} k_{h}\left(k^{h}-k_{p}\right)^{2}\left(\tilde{m}-s_{p}^{2}\right)+4 k^{h} k_{h}\left(k^{p}+k_{p}\right)^{2}\left(s_{h}^{2}\right.\right.$

$$
-\tilde{m})] / \mathcal{J}_{0}^{2} \text {. }
$$

Here we also used $\varsigma_{h}=\tilde{m} / s_{p}$ and $\varsigma_{p}^{2}<\tilde{m}<s_{h}^{2}$.
[1] G. B. Partridge, W. Li, R. I. Kamar, Y. A. Liao, and R. G. Hulet, Science 311, 503 (2006).

[2] G. B. Partridge, W. Li, Y. A. Liao, R. G. Hulet, M. Haque, and H. T. C. Stoof, Phys. Rev. Lett. 97, 190407 (2006).

[3] M. W. Zwierlein, A. Schirotzek, C. H. Schunck, and W. Ketterle, Science 311, 492 (2006).

[4] M. W. Zwierlein, C. H. Schunck, A. Schirotzek, and W. Ketterle, Nature (London) 442, 54 (2006).

[5] Y. Shin, M. W. Zwierlein, C. H. Schunck, A. Schirotzek, and W. Ketterle, Phys. Rev. Lett. 97, 030401 (2006).

[6] C. H. Schunck, Y. Shin, A. Schirotzek, M. W. Zwierlein, and W. Ketterle, Science 316, 867 (2007).

[7] Y. Shin, C. H. Schunck, A. Schirotzek, and W. Ketterle, Nature (London) 451, 689 (2008).

[8] M. Houbiers, R. Ferwerda, H. T. C. Stoof, W. I. McAlexander, C. A. Sackett, and R. G. Hulet, Phys. Rev. A 56, 4864 (1997).

[9] D. E. Sheehy and L. Radzihovsky, Ann. Phys. (N.Y.) 322, 1790 (2007).

[10] S. Giorgini, L. P. Pitaevskii, and S. Stringari, Rev. Mod. Phys. 80, 1215 (2008).
[11] W. Ketterle and M. W. Zwierlein, Riv. Nuovo Cimento 31, 247 (2008).

[12] D. E. Sheehy and L. Radzihovsky, Phys. Rev. Lett. 96, 060401 (2006).

[13] Q. Chen, J. Stajic, S. Tan, and K. Levin, Phys. Rep. 412, 1 (2005).

[14] F. Chevy, Phys. Rev. Lett. 96, 130401 (2006).

[15] K. B. Gubbels and H. T. C. Stoof, Phys. Rev. Lett. 100, 140407 (2008).

[16] K. B. Gubbels, M. W. J. Romans, and H. T. C. Stoof, Phys. Rev. Lett. 97, 210402 (2006).

[17] P. F. Bedaque, H. Caldas, and G. Rupak, Phys. Rev. Lett. 91, 247002 (2003).

[18] M. M. Parish, F. M. Marchetti, A. Lamacraft, and B. D. Simons, Nat. Phys. 3, 124 (2007).

[19] M. Haque and H. T. C. Stoof, Phys. Rev. A 74, 011602(R) (2006).

[20] T. N. De Silva and E. J. Mueller, Phys. Rev. Lett. 97, 070402 (2006).

[21] A. Lazarides and B. Van Schaeybroeck, Phys. Rev. A 77, 
041602(R) (2008).

[22] C. Lobo, A. Recati, S. Giorgini, and S. Stringari, Phys. Rev. Lett. 97, 200403 (2006).

[23] J. Tempere, S. N. Klimin, and J. T. Devreese, Phys. Rev. A 78, 023626 (2008).

[24] B. Van Schaeybroeck and A. Lazarides, Phys. Rev. Lett. 98, 170402 (2007).

[25] P. G. de Gennes, Superconductivity of Metals and Alloys (Addison-Wesley, Reading, MA, 1989).

[26] A. L. Fetter and J. D. Walecka, Quantum Theory of ManyParticle Systems (McGraw Hill, Boston, 1971).

[27] G. E. Blonder, M. Tinkham, and T. M. Klapwijk, Phys. Rev. B 25, 4515 (1982).

[28] Due to the momentum-independent interaction strength, one must cancel the ultraviolet divergencies originally appearing in the integral. In the presence of a HF term this was treated in Refs. [8,29,30,56-60] for homogeneous systems.

[29] T. Papenbrock and G. F. Bertsch, Phys. Rev. C 59, 2052 (1999).

[30] W.-C. Su, Phys. Rev. A 74, 063627 (2006).

[31] I. S. Gradshteyn and I. M. Ryzhik, Table of Integrals, Series and Products, 6th ed. (Academic Press, San Diego, 1980).

[32] M. Marini, F. Pistolesi, and G. C. Strinati, Eur. Phys. J. B 1, 151 (1998)

[33] A. Bulgac, Phys. Rev. A 76, 040502(R) (2007).

[34] G. Bertaina and S. Giorgini, Phys. Rev. A 79, 013616 (2009).

[35] M. Haque and H. T. C. Stoof, Phys. Rev. Lett. 98, 260406 (2007).

[36] R. Haussmann and W. Zwerger, Phys. Rev. A 78, 063602 (2008).

[37] A. Recati, C. Lobo, and S. Stringari, Phys. Rev. A 78, 023633 (2008).

[38] A. M. Clogston, Phys. Rev. Lett. 9, 266 (1962).

[39] A. F. Andreev, Zh. Eksp. Teor. Fiz. 46, 1823 (1964); Sov. Phys. JETP 19, 1228 (1964).
[40] M. Urban, Phys. Rev. A 75, 053607 (2007).

[41] I. Zapata and F. Sols, e-print arXiv:0804.4631, Phys. Rev. Lett. (to be published).

[42] S. D. Huber and G. Blatter, e-print arXiv:0807.3173, Phys. Rev. B (to be published).

[43] J. Demers and A. Griffin, Can. J. Phys. 49, 285 (1971).

[44] L. D. Landau and E. M. Lifshitz, Quantum Mechanics (Nonrelativistic Theory) (Pergamon, Oxford, 1977).

[45] A. F. Andreev, Sov. Phys. JETP 49, 655 (1966); 22, 455 (1966).

[46] Note that $h_{S}$ is eliminated using the coexistence condition Eq. (10).

[47] K. Huang, Statistical mechanics, 1st ed. (Wiley, New York, 1963).

[48] M. Tinkham, Introduction to Superconductivity, 2nd ed. (McGraw-Hill, New York, 1996).

[49] W. L. McMillan, Phys. Rev. 175, 559 (1968).

[50] Y. Tanaka and M. Tsukada, Phys. Rev. B 42, 2066 (1990).

[51] P. C. van Son, H. van Kempen, and P. Wyder, Phys. Rev. B 37, 5015 (1988).

[52] G. Kieselmann, Phys. Rev. B 35, 6762 (1987).

[53] Y. Nagato, K. Nagai, and J. Hara, J. Low Temp. Phys. 93, 33 (1993).

[54] A. A. Abrikosov, Fundamentals of the Theory of Metals (Elsevier, New York, 1988).

[55] J. Stajic, J. N. Milstein, Q. Chen, M. L. Chiofalo, M. J. Holland, and K. Levin, Phys. Rev. A 69, 063610 (2004).

[56] J. Tempère and J. T. Devreese, J. Phys. B 39, S57 (2006).

[57] Y. Ohashi and A. Griffin, Phys. Rev. A 72, 013601 (2005).

[58] M. Randeria, in Bose Einstein Condensation, edited by Griffin, Stroke, and Stringari (Cambridge University Press, Cambridge, 1995).

[59] A. Bulgac and Y. Yu, Phys. Rev. Lett. 88, 042504 (2002).

[60] M. Grasso and M. Urban, Phys. Rev. A 68, 033610 (2003). 